\title{
THE COMPLEXITY OF RELIABILITY COMPUTATIONS IN PLANAR AND ACYCLIC GRAPHS*
}

\author{
J. SCOTT PROVAN $\dagger$
}

\begin{abstract}
We show that the problem of computing source-sink reliability is NP-hard, in fact \# Pcomplete, even for undirected and acyclic directed source-sink planar graphs having vertex degree at most three. Thus the source-sink reliability problem is unlikely to have an efficient algorithm, even when the graph can be laid out on a rectilinear grid.
\end{abstract}

Key words. reliability, complexity, planar graph, acyclic graph, NP-hard, \# P-complete

1. Introduction. Connectedness reliability problems on graphs have long constituted a class of computationally intractable problems. Virtually all such problems have been shown to be NP-hard (actually \# P-complete) for general graphs [2], [10], [11], [16]. Work has therefore concentrated on finding significant special classes of graphs for which polynomial algorithms do exist for computing network reliability. This has culminated in methods for computing fairly general reliability measures in directed and undirected series-parallel graphs [1], [13]. There are three important classes of graphs for which complexity results have not been obtained, namely, planar graphs, acyclic graphs, and graphs which have bounded vertex degree. There is, moreover, compelling evidence to suggest that reliability problems for these graphs might be computationally easier than for general graphs (see [3], [7], [9], [10], [14]). We show in this paper, however, that the problem of computing source-sink reliability is \# P-complete, even for undirected and acyclic directed source sink planar graphs having vertex degree at most three.

2. Preliminaries. Let $G=(V, E)$ be a graph (directed or undirected) with vertex set $V$ of cardinality $m$ and edge set $E$ of cardinality $n$. The degree of a vertex is the number of edges adjacent to that vertex. Two vertices $s$ and $t$ are distinguished as the source and sink vertices, respectively. The graph $G$ is called source-sink planar, or simply $(s, t)$-planar, if it has a planar representation with $s$ and $t$ on the boundary. Now suppose edges in $G$ fail independently, each edge failing with the same probability $1-p, 0 \leqq p \leqq 1$. For the purposes of this paper, we take $p$ to be rational. Then the $(s, t)$-connectedness reliability of $G, R(G, s, t ; p)$, is the probability that there is at least one path of operating edges from $s$ to $t$, where the path is taken to be directed when $G$ is directed. This probability can be written as

$$
R(G, s, t ; p)=\sum_{H \in \mathscr{H}} p^{|H|}(1-p)^{n-|H|}
$$

where $\mathscr{H}$ is the collection of sets of edges which contain at least one $(s, t)$-path. The problem of computing $R(G, s, t ; p)$ has been shown to be \# P-complete for undirected graphs by Valiant [16], and for directed acyclic graphs in [10]. In neither case, however, are the networks planar, nor are the vertex degrees bounded. The two problems considered in this paper are

P3ST. The problem of computing $R(G, s, t ; p)$ when $G$ is an undirected $(s, t)$ planar graph with each vertex having degree at most three;

\footnotetext{
* Received by the editors April 17, 1984, and in revised form January 2, 1985. This research was supported in part by the Air Force Office of Scientific Research under contract AFOSR-84-0140.

$\dagger$ Operations Research and Systems Analysis, University of North Carolina, Chapel Hill, North Carolina 27514.
} 
PA3ST. The problem of computing $R(G, s, t ; p)$ when $G$ is an acyclic directed

$(s, t)$-planar graph with each vertex having degree at most three.

It is at present unknown whether there exists a polynomial algorithm to solve either of these problems.

We explore the complexity of the problems P3ST and PA3ST in the manner proposed by Valiant [16]. We assume that the reader is familiar with the notions of NP and NP-complete problems; see [4] for an excellent account of these concepts and their relationship to \#P-completeness. Fix input alphabet $\Sigma$ and denote by $\Sigma^{*}$ the corresponding collection of (finite) strings. We assume $\Sigma^{*}$ contains a representation of $Z_{+}=\{0,1, \cdots\}$. Define the class \#P to consist of those functions $f: \Sigma^{*} \rightarrow Z_{+}$which can be computed by counting the number of accepting computations of some nondeterministic Turing machine of polynomial time complexity. For function $f: \Sigma^{*} \rightarrow Z_{+}$, define a f-oracle Turing machine to be a Turing machine, together with an additional input and output tape, which at any time during a computation can write string $\sigma$ on the input tape and in one step receive $f(\sigma)$ on the output tape. A function $g: \Sigma^{*} \rightarrow Z_{+}$is polynomially reducible to $f(g \propto f)$ if there exists a polynomial time complexity $f$-oracle Turing machine which computes $g$. A function $f$ is called \#P-complete if (a) $f$ is in \# $\mathrm{P}$ and (b) every function $g$ in \# $\mathrm{P}$ is polynomially reducible to $f$.

Roughly speaking, the \# P-complete problems are those which are polynomially equivalent to the counting problems associated with many NP-complete problems-for example, counting the number of Hamiltonian circuits in a graph. They are therefore at least as hard as NP-complete problems, and so it is unlikely that a polynomial algorithm exists to solve these problems. It should be noted that P3ST and P3AST are technically not \# $\mathrm{P}$ problems, since, among other things they compute rational numbers rather then integers. However, it will follow from subsequent discussion (specifically Corollary 1) that in both P3ST and PA3ST the problem of computing $R$ can be reduced to that of computing $2^{n} R(G, s, t ; 1 / 2)=|\mathscr{H}|=$ the number of sets of edges which admit a path from $s$ to $t$. This function is clearly in \# $\mathrm{P}$, since such edge sets are easily recognizable.

We will show that both P3ST and PA3ST are \# P-complete problems. This result is somewhat surprising, since several \# P-complete enumeration problems associated with $(s, t)$-connectness-i.e., the number of $(s, t)$-paths of any given length and the number of minimum cardinality $(s, t)$-cuts-become polynomially computable when restricted to planar graphs (in the case of minimum cuts) or acyclic graphs (in the case of paths) [3]. The planarity and bounded vertex degree in P3ST and PA3ST also mean that the problem of computing $R(G, s, t ; p)$ is \#P-complete even when $G$ can be embedded in a rectangular grid with $s$ and $t$ on the perimeter (see [5, Thm. 1] for details).

As a starting point for the results in this paper, we present two known \# P-complete functional evaluation problems.

I. Number of Hamiltonian circuits in a planar cubic graph (\# HCPC)

Given: planar undirected graph with each vertex of degree three;

Find: the number of closed simple paths going through every vertex of $G$.

II. Acyclic $(s, t)$-connectedness reliability (AST)

Given: acyclic graph $G$, source $s$, sink $t$, and rational probability $p$.

Find: $R(G, s, t ; p)$,

As with P3ST and PA3ST, one can think of AST as computing $2^{n} R\left(G, s, t ; \frac{1}{2}\right)=|\mathscr{C}|$ so as to make it a member of \#P. The problem AST was shown to be \#P-complete in [10]. The problem \# HCPC has essentially been shown to be \#P-complete in [6]. Specifically, in the construction for the $m$ clause, 3-conjunctive normal form expression 
$F$ in that paper, if the "required-edge" graph is placed into the successive figures as oriented, then the resulting graph has exactly $\left(8^{7} \cdot 18\right)^{m} \cdot 8^{6 a} \cdot 8^{b} \cdot 36$ Hamiltonian circuits for each assignment satisfying $F$, where $a$ is the number of "crossing exclusive-or" graphs and $b$ is the number of "noncrossing exclusive-or" graphs added in the final construction. Since the number of satisfying assignments for a 3-conjunctive normal form expression is known to be \# P-complete [15], then \# HCPC is also \# P-complete.

We next present a key formula used to prove both of the main results of this paper. It is due to Satyanarayana and Prabhakar ([12, eq. (3)], with a correct proof in [17]), and is restated here as it applies to P3ST and PA3ST. For directed graph $G$ and specified vertices $s$ and $t$, define an $(s, t)$-subgraph of $G$ to be an acyclic subgraph $H$ of $G$ having the property that every edge of $H$ lies in at least one path in $H$ from $s$ to $t$. Equivalently, an $(s, t)$-subgraph can be characterized as an acyclic subgraph $H$ such that $s$ is the only vertex with no edges of $H$ pointing into it and $t$ is the only vertex with no edges of $H$ pointing out of it. Satyanarayana and Prabhakar showed that the $(s, t)$-connectedness reliability for $G$ can be written

$$
R(G, s, t ; p)=\sum_{i, j} \sum_{H \in \mathscr{K}_{i j}}(-1)^{j-i+1} p^{j}
$$

where $\mathscr{H}_{i j}$ is the set of $(s, t)$-subgraphs $H$ with $i$ vertices and $j$ edges. This formula can be applied as well when $G$ is undirected. To do this, we first construct directed graph $G^{\prime}$ by replacing each undirected edge of $G$ by two oppositely directed edges, each with probability $p$. Then, as proved in [2, Thm. 2], $R\left(G^{\prime}, s, t ; p\right)$ is equal to $R(G, s, t ; p)$. The $(s, t)$-subgraphs of $G$ now correspond to acyclic orientations of undirected subgraphs of $G$ for which every edge is in at least one $(s, t)$-path, so that an $(s, t)$-subgraph for an undirected graph will always refer to the corresponding oriented subgraph. With this modification, equation (1) holds also when $G$ is undirected.

Finally, we present three general lemmas. The first is due to Valiant [16, Fact 5]. Define the size of a rational number $r$ to be the total number of (binary) digits in the numerator and denominator, when $r$ is presented as a fraction in lowest terms.

LEMMA 1. If $g(x)$ is an nth degree polynomial with rational coefficients and its value is known at each of the distinct rational points $x_{1}, \cdots, x_{n+1}$ each of size at most $d$, then the coefficients of $g$ can be deduced in time polynomial in $n, d$, and the maximum size of the values of $g\left(x_{i}\right)$.

To give the second lemma, we need some additional notation. Let $G, s, t$, and $p$ be given, and let $S$ be a subset of edges of $G$. For $l=0, \cdots,|S|$ define

$$
R^{l}(G, s, t, S ; p)=\sum_{i, j} \sum_{H \in \mathscr{H}_{i j}^{l}}(-1)^{j-i+1} p^{j}
$$

where $\mathscr{H}_{i j}^{l}$ is the set of $(s, t)$-subgraphs of $G$ with $i$ vertices, $j$ edges of $E-S$, and $l$ edges of $S$.

LEMmA 2. $R^{l} \propto R$, even when restricted to graphs which are $(s, t)$-planar, acyclic, or with vertex degree at most three.

Proof. Let the arguments $G, s, t, S$ and $p$ be given, with $m$ the number of vertices of $G, n$ the number of edges of $G, k$ the cardinality of $S$, and $d$ the size of $p$. For $r=0, \cdots, k$ construct the graph $G_{r}$ by replacing each edge $e=(u, v)$ of $S$ by the path of $r+1$ edges $\left(u, u_{e, 1}\right)\left(u_{e, 1}, u_{e, 2}\right), \cdots,\left(u_{e, r} v\right)$, where $u_{e, 1}, \cdots, u_{e, r}$ are new vertices. This graph has $m+k r$ vertices and $n+k(r+1)$ edges, and is planar, acyclic, or with vertex degree at most three, respectively, if $G$ is. Further, an $(s, t)$-subgraph in $G_{r}$ corresponds to an $(s, t)$-subgraph of $G$ with each (oriented) edge of $S$ replaced by the appropriate 
(oriented) path. Equation (1) now becomes

$$
\begin{aligned}
R\left(G_{r}, s, t ; p\right) & =\sum_{l=0}^{k} \sum_{i, j} \sum_{H \in \mathscr{H}_{i j}^{l}}(-1)^{j+l(r+1)-(i+l r)+1} p^{j+l(r+1)} \\
& =\sum_{l=0}^{k}\left(-p^{r+1}\right)^{l} R^{l}(G, s, t, S ; p) .
\end{aligned}
$$

This is a polynomial in $-p^{r+1}$. The coefficients $R^{l}(G, s, t, S ; p)$ consist of sums of at most $2^{2 n}$ terms (an upper bound on the number of (oriented) $(s, t)$-subgraphs of $G$ ) with size at most $n d$, so that the size of $R^{l}(G, s, t, S ; p)$ is bounded by $n(d+2)$. Similarly, the size of each $R\left(G_{r}, s, t ; p\right)$ is bounded by $(n+k(r+1))(d+2)$. Using Lemma 1 we can compute the $k+1$ coefficients $R^{l}(G, s, t, S ; p), l=0, \cdots, k$, from the $k+1$ values $R\left(G_{r}, s, t ; p\right), r=0, \cdots, k$, in time polynomial $m, n$, and $d$. This proves the lemma.

Note that the reduction in Lemma 1 can be performed when $p$ is fixed at any value other than 0 or 1 , specifically for $p=1 / 2$. Furthermore, if we consider the case when $S=E$, then for $l=0, \cdots, n$ we have

$$
R^{l}(G, s, t, E ; p)=\sum_{i} \sum_{H \in \mathscr{C}_{i 0}^{l}}(-1)^{i-1}
$$

where $\mathscr{H}_{i 0}^{l}$ is the set of $(s, t)$-subgraphs of $G$ with $i$ vertices and $l$ edges, i.e. $\mathscr{H}_{i 0}^{l}=\mathscr{K}_{i l}$. But now we can write

$$
R(G, s, t ; p)=\sum_{l}(-p)^{l} R^{l}(G, s, t, E ; p) .
$$

From the above discussion we obtain the following corollary.

COROllary 1. $R \propto R(\cdot ; p=1 / 2)$, even when restricted to graphs which are $(s, t)$ planar, acyclic, or with vertex degree at most three.

As stated when defining P3ST, PA3ST, and AST, then, there is no loss of generality in considering the \# P-problem of computing $|\mathscr{H}|$ rather than the reliability problem of computing $R$.

Lemma 2 provides a second useful corollary. Let $G, s, t$, and $S$ be as in Lemma 2 , and for $l=0, \cdots,|S|, k=0, \cdots,|E-S|$ define

$$
R^{l k}(G, s, t, S)=\sum_{i} \sum_{H \in \mathscr{C}_{i k}^{l}}(-1)^{i-1}
$$

where $\mathscr{H}_{i k}^{l}$ is as defined for $R^{l}$. By applying Lemma 2 twice, once to $S$ and once to $E-S$, we have the following result:

COROLlARY 2. $R^{l k} \propto R$, even when restricted to graphs which are $(s, t)$-planar, acyclic, or with vertex degree at most three.

Again, the restriction to $p=1 / 2$ provides no loss of generality.

3. \#P-complete results. We first prove the result for the undirected case.

THEOREM 1. P3ST is \# P-complete, in particular, \# HCPC $\propto \mathrm{P} 3 \mathrm{ST}$.

Proof. Let $G=(V, E)$ be a planar undirected cubic graph with $m$ vertices and $n$ edges. Chose any edge $(u, v)$, and form the graph $G^{\prime}=\left(V^{\prime}, E^{\prime}\right)$ by replacing $(u, v)$ by the two edges $(s, u)$ and $(v, t)$, where $s$ and $t$ are new vertices. The graph $G^{\prime}$ is clearly $(s, t)$-planar. Now form the graph $G^{\prime \prime}=\left(V^{\prime \prime}, E^{\prime \prime}\right)$ by replacing each (degree 3$)$ vertex except $s$ and $t$ with a triangle as shown in Fig. 1. Then $\left|V^{\prime \prime}\right|=3 m+2$ and $\left|E^{\prime \prime}\right|=$ $n+3 m+1$, and each edge of $E^{\prime}$ can be identified with the appropriate edge of $E^{\prime \prime}$. Let $S$ be the set of edges of $E^{\prime \prime}$ associated with $E^{\prime}$ so that $T=E^{\prime \prime}-S$ is the set of triangle 


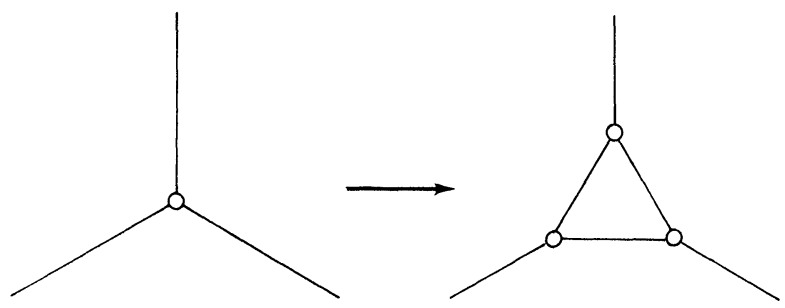

FIG. 1

edges. By applying Corollary 2, we conclude that the function

$$
R^{m+1, m}\left(G^{\prime \prime}, s, t, S\right)=\sum_{i, j} \sum_{H \in \mathscr{K}_{i, m}^{m+1}}(-1)^{i-1}
$$

-where $\mathscr{H}_{i, m}^{m+1}$ is the set of all oriented $(s, t)$-subgraphs of $G^{\prime \prime}$ with $i$ vertices, $m+1$ edges $S$ and $m$ edges of $T$-is polynomially reducible to P3ST. Now for any $H$ in $\mathscr{H}_{i, m}^{m+1}$, consider the oriented subgraph $H^{\prime}$ of $G^{\prime}$ corresponding to the $m+1$ edges of $H$ in $S$. These must comprise an $(s, t)$-subgraph of $G^{\prime}$, which means that each vertex in $H^{\prime}$ except $s$ and $t$ must have degree either 2 or 3 . Let $V_{i}$ be the set of vertices of $H^{\prime}$ of degree $i, i=2,3$. Again, since $H$ is an $(s, t)$-subgraph then corresponding to each vertex in $V_{2}$ there must be at least one edge of $T \cap H$ and corresponding to each vertex in $V_{3}$ there must be at least two edges of $T \cap H$. Let $k$ be the total number of edges of $T \cap H$ corresponding to vertices in $V_{3}$, so that $m-k$ is the total number of edges of $T \cap H$ corresponding to vertices in $V_{2}$. By summing the degrees of vertices in $H^{\prime}$, we have

$$
2(m+1)=2\left|V_{2}\right|+3\left|V_{3}\right|+2 \leqq 2(m-k)+3 k / 2+2=2(m+1)-k / 2
$$

implying that $k=0$. This means that $H^{\prime}$ must be a Hamiltonian path in $G$, and that $H$ is obtained from $H^{\prime}$ by adding the unique set of $m$ edges of $T$ connecting the appropriate edges of $S \cap H$ at each vertex. Thus each $H$ has exactly $2 m+2$ vertices and corresponds in one-to-one fashion with the Hamiltonian paths from $s$ to $t$ in $G$. It follows that

$$
R^{m+1, m}\left(G^{\prime \prime}, s, t, S\right)=-\left|\mathscr{H}_{2 m+2, m}^{m+1}\right|
$$

and we therefore obtain the number of Hamiltonian paths from $s$ to $t$ in $G^{\prime}$, which corresponds to the number of Hamiltonian circuits in $G^{\prime}$ containing the edge $(u, v)$. In a similar manner, we can obtain the number of Hamiltonain circuits containing each of the other two edges of $G$ adjacent to $v$, and the sum of these three values is exactly twice the number of Hamiltonian circuits in $G$. This completes the proof of the theorem.

To prove that PA3ST is \# P-complete, we provide an intermediate reduction. Define a directed $(s, t)$-planar graph $G$ to be contiguously directed if it has a planar presentation for which each vertex $v$ has its adjacent edges arranged so that in a clockwise sweep around $v$ the edges into $v$ and the edges out of $v$ lie in two unbroken sequences. Define the problem PCAST to be that of computing $R(G, s, t ; p)$ in an $(s, t)$-planar contiguously directed acyclic graph. We note that Lemma 2 and its corollaries can easily be seen to include the restriction to contiguously directed graphs.

THEOREM 2. AST $\propto$ PCAST.

Proof. Suppose we are given acyclic directed graph $G=(V, E)$ with $m$ vertices, $n$ edges, source $s$, and sink $t$. Since $G$ is acyclic, it follows that we can number the 
vertices of $G v_{1}, \cdots, v_{m}$ such that $\left(v_{i}, v_{j}\right) \in E$ if and only if $i<j$. We can therefore place the vertices of $G$ in the plane, representing the edges as straight lines, in such a way that each vertex $v_{i}$ has $x$-coordinate $i, i=1, \cdots, m$, no three edges cross at the same point, and no edge crosses a nonadjacent vertex. Further, this construction can be done in polynomial time. This realization of $G$ has the properties that no simple path can cross itself in the realization, and that the number of edge crossings is $c \leqq n^{2}$. Now construct the graph $\bar{G}=(\bar{V}, \bar{E})$ by successively replacing each pair of crossing edges in $G$ by the planar subgraph shown in Fig. 2. We define the set $S$ to comprise the collection of heavy-lined edges as shown in the figure and we set $T=\bar{E}-S$. Then $\bar{G}$ is planar with $m+6 c$ vertices, $6 c$ edges in $S$ and $n+3 c$ edges in $T$. Further, with the given realization of $G$ it follows that $\bar{G}$ remains acyclic, and that $\bar{G}$ is contiguously directed since all edges come into a vertex from the left and leave to the right.

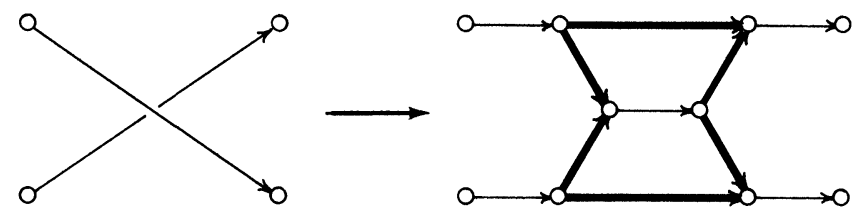

FIG. 2

Now for $l=1, \cdots, 6 c, k=1, \cdots, n+3 c$, define $R^{l k}$ as in (2):

$$
R^{l k}(\bar{G}, s, t, S)=\sum_{i=0}^{m} \sum_{H \in \overline{\mathscr{H}}_{i k}^{l}}(-1)^{i-1}
$$

where $\overline{\mathscr{H}}_{i k}^{l}$ is the number of $(s, t)$-subgraphs of $\bar{G}$ with $i$ vertices, $l$ edges of $S$ and $k$ edges of $T$. It follows from Corollary 2 that $R^{l k}(\bar{G}, s, t, S)$ is reducible to $R$ with $R$ restricted to $(s, t)$-planar contiguously directed acyclic graphs. The theorem is an immediate consequence of Corollary 2 and the following claim.

Claim.

$$
R(G, s, t ; 1 / 2)=\sum_{l, k}(-1)^{l+k} R^{l k}(\bar{G}, s, t, S)(1 / 2)^{k-l} .
$$

Proof of claim. We first rewrite (3) as

$$
R(G, s, t ; 1 / 2)=\sum_{i, l, k} \sum_{H \in \mathscr{\mathscr { H }}_{i k}^{l}}(-1)^{l+k-i+1}(1 / 2)^{k-l} .
$$

We prove the claim by induction on the number $r$ of replacements of the type shown in Fig. 2. The case $r=0$ follows from (1). Now suppose that (4) holds after $r$ replacements with $\bar{G}$ the resulting graph. Suppose we replace the pair $(u, v),(w, z)$ of crossing edges by the appropriate subgraph $F$ to form graph $\bar{G}^{\prime}$. Consider an $(s, t)$-subgraph $H^{\prime}$ of $\bar{G}^{\prime}$. This subgraph falls into one of 10 classes, depending on which of the four edges of $F$ adjacent to $u, v, w$, and $z$ appear in $H^{\prime}$. By symmetry we need to consider only five classes, namely class 1 -in which none of these four edges appears-or one of the four classes shown in Fig. 3. For each class, consider the possible configurations of internal edges of $F$ which could form an $(s, t)$-subgraph with the given outside edges. By summing $(-1)^{l^{\prime}+k^{\prime}-i^{\prime}+1}(1 / 2)^{k^{\prime}-l^{\prime}}$ over all configurations in a class-where $i^{\prime}$, $k^{\prime}$, and $l^{\prime}$ are, respectively, the number of vertices, number of edges of $S$, and number of edges of $T$ appearing in the configuration (including outside edges and vertices) -we get:

(i) the sum over all configurations in class 2 and 3 is 0 ; 

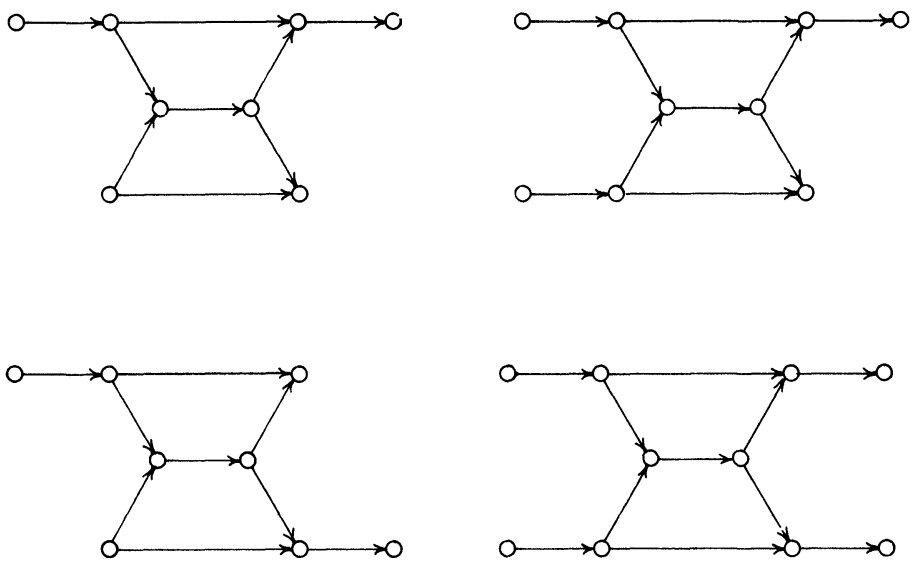

FIG. 3

(ii) the sum over all configurations in class 4 is $\frac{1}{2}$;

(iii) the sum over all configurations in class 5 is $-\frac{1}{4}$.

Now consider an $(s, t)$-subgraph $H$ in $\bar{G}$. Then $H$ falls into one of four classes, depending on which subset of the edges $(u, v)$ and $(w, z)$ appear in $H$. If $H$ contains neither $(u, v)$ nor $(w, z)$ then $H$ corresponds to exactly one subgraph of $\bar{G}^{\prime}$, which is in class 1 ; if $H$ contains exactly one of the two edges, say $(u, v)$, then $H$ corresponds to the class of $(s, t)$-subgraphs of $\bar{G}^{\prime}$ comprised of the edges $H-F$ along with the set of class 4 configurations of $F$; and if $H$ contains both of the edges $(u, v)$ and $(w, z)$, then $H$ corresponds to the class of $(s, t)$-subgraphs of $\bar{G}^{\prime}$ comprised of the edges $H-F$ along with the set of class 5 configurations of $F$. In each case, the net contribution to (4) of configurations in classes 1,4 , and 5 is exactly equal to contribution of the edges $(u, v)$ and $(w, z)$, and the net contribution of configurations in classes 2 and 3 is zero. It follows that (4) continues to hold for the graph $\bar{G}^{\prime}$, and the claim follows by induction. This completes the proof of the theorem.

We can now present the result for the directed case.

TheOREM 3. PA3ST is \# P-complete, in particular PCAST $\propto$ PA3ST.

Proof. Let $G=(V, E)$ be an $(s, t)$-planar acyclic contiguously directed graph with $m$ vertices and $n$ edges. For each vertex $v$, let

$$
\left(u_{1}(v), v\right),\left(u_{2}(v), v\right), \cdots,\left(u_{l(v)}(v), v\right),\left(v, u_{l(v)+1}(v)\right), \cdots,\left(v, u_{k(v)}(v)\right)
$$

be the edges adjacent to $v$, listed in clockwise order. Now define the directed graph $G^{\prime}=\left(V^{\prime}, E^{\prime}\right)$ with vertex set

$$
V^{\prime}=\left\{w_{1}(v), \cdots, w_{k(v)}(v) ; v \in V\right\}
$$

and edge set $E^{\prime}=S \cup T$ with

$$
\begin{aligned}
& S=\left\{\left(w_{1}(v), w_{2}(v)\right), \cdots,\left(w_{k(v)-1}(v), w_{k(v)}(v)\right): v \in V\right\} \\
& T=\left\{\left(w_{i}(u), w_{j}(v)\right):(u, v) \in E \text { with } u=u_{i}(v) \text { and } v=u_{j}(u)\right\}
\end{aligned}
$$

The edges can be positioned as shown in Fig. 4. Finally, set $s^{\prime}=w_{1}(s)$ and $t^{\prime}=w_{k(t)}(t)$. It is clear that $G^{\prime}$ is $\left(s^{\prime}, t^{\prime}\right)$-planar and acyclic, that it has at most three edges adjacent to each vertex, and that $\left|V^{\prime}\right|=2 n,|S|=2 n-m$, and $|T|=n$. Further, there is a one-to-one correspondence between the $(s, t)$-subgraphs of $G$ and the $\left(s^{\prime}, t^{\prime}\right)$-subgraphs of $G^{\prime}$, 

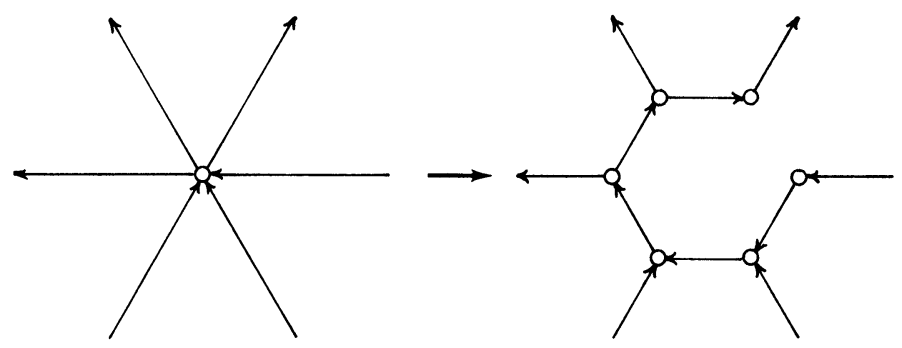

FIG. 4

obtained by associating with each $(s, t)$-subgraph $H$ of $G$ the subgraph $H^{\prime}$ of $G^{\prime}$ with edge set $A \cup B$ where

$A=\left\{\left(w_{i}(u), w_{j}(v)\right):(u, v) \in H\right.$ with $\left.u=u_{i}(v), v=u_{j}(u)\right\} \subseteq T$,

$B=\left\{\begin{array}{l}\left(w_{i}(v), w_{i+1}(v)\right),\left(w_{i+1}(v), w_{i+2}(v)\right), \cdots,\left(w_{j-1}(v), w_{j}(v)\right): v \in H, \\ i=\left\{\begin{array}{ll}1, & v=s, \\ \min \left\{p:\left(u_{p}(v), v\right) \in H\right\}, & v \neq s,\end{array} \quad j=\left\{\begin{array}{ll}k(v), & v=t, \\ \max \left\{p:\left(v, u_{p}(v)\right) \in H\right\}, v \neq t\end{array}\right\} \subseteq S\right.\end{array}\right.$

so that $H^{\prime}$ has exactly $|B|$ more vertices than $H$. From Lemma 2 we get that $R^{l}\left(G^{\prime}, s^{\prime}, t^{\prime}, S ; p\right)$ is polynomially reducible to $R$, with $R$ restricted to $(s, t)$-planar, acyclic graphs with vertex degree at most 3 . But now

$$
\begin{aligned}
R(G, s, t ; p) & =\sum_{i, j} \sum_{H \in \mathscr{H}_{i j}}(-1)^{j-i+1} p^{j} \\
& =\sum_{l}(-1)^{l} R^{l}\left(G^{\prime}, s^{\prime}, t^{\prime}, S ; p\right)
\end{aligned}
$$

and the theorem follows.

4. Conclusion. We have shown in this paper that planarity, acyclicness, and bounded vertex degree, even taken together, are not sufficient to keep the problem of computing $(s, t)$-connectedness reliability from being NP-hard. This is true even though several counting problems associated with $(s, t)$-connectedness reliability become polynomial when restricted to these classes of graphs. A second important connectedness reliability problem for which the same type of analysis could be performed is the source-to-all, or connectedness, reliability problem. This is the problem of computing for a graph $G$-under the same edge failure distribution used in this paper-the probability that a given source vertex can reach all other vertices of $G$ through paths of operating edges. For this measure, the acyclic property alone is sufficient to construct a polynomial evaluation algorithm (see [3, Thm. 4(v)]). Whether planarity alone is sufficient remains an open problem, although again several related NP-hard problems can be solved in polynomial time on planar graphs (see [3, Thm. 4(iv)], [10], and [9, Corollary 2.7 and succeeding discussion]). Further, the class of planar graphs for which this reliability can be computed efficiently has been extended slightly from seriesparallel graphs to include "Cube-Free" graphs [8]. The problem for planar graphs with bounded vertex degree also remains open.

Acknowledgment. The author would like to thank Mark Jerrum for comments which significantly improved the results and presentation of this paper. 


\section{REFERENCES}

[1] A. Agrawal And A. Satyanarayana, $A n O(|E|)$-time algorithm for computing the reliability of a class of directed networks, Oper. Res., 32 (1984), pp. 493-515.

[2] M. O. BALL, The complexity of network reliability computations, Networks, 10 (1977), pp. 153-165.

[3] M. O. Ball AND J. S. Provan, Calculating bounds on reachability and connectedness in stochastic networks, Networks, 13 (1983), pp. 253-278.

[4] M. R. Garey AND D. S. Johnson, Computers and Intractibility: A Guide to the Theory of NPCompleteness, W. H. Freeman, San Francisco, CA, 1979.

[5] - The rectilinear Steiner tree problem is NP-complete, SIAM J. Appl. Math., 32 (1977), pp. 826-834.

[6] M. R. GAREY, D. S. Johnson AND R. E. TARJAN, The planar Hamiltonian circuit problem is Np-complete, this Journal, 5 (1976), pp. 704-714.

[7] M. G. LuBY, Monte-carlo methods for estimating system reliability, Tech. Rep. 84/168, Computer Science Division, Univ. California, Berkeley, CA, 1982, Chapter 6.

[8] T. Politof AND A. Satyanarayana, A linear time algorithm to compute the reliability of planar cube-free graphs, Tech. Rept., Dept. Electrical Engineering and Computer Science, Stevens Institute of Technology, Hoboken, NJ, 1985.

[9] J. S. Provan, Polyhedral combinatorics and network reliability, Math. Oper. Res., to appear.

[10] J. S. PROVAN AND M. O. BALL, The complexity of counting cuts and computing the probability that a graph is connected, this Journal, 12 (1983), pp. 777-788.

[11] A. RosenthaL, Computing the reliability of complex networks, SIAM J. Appl. Math., 32 (1977), pp. 384-393.

[12] A. Satyanarayana AND A. PRABhakar, New topological formula and rapid algorithm for reliability analysis of complex networks, IEEE Trans. Reliability, R-27 (1978), pp. 82-100.

[13] A. Satyanarayana AND R. K. Wood, Polygon-to-chain reductions and network reliability, this Journal, 14 (1985), pp. 818-832.

[14] A. W. SHOGAN, Sequential bounding of the reliability of a stochastic network, Oper. Res., 24 (1976), pp. 1027-1044.

[15] J. SIMON, On some central problems in computational complexity, Ph.D. Thesis (Tech. Rep. TR75-224), Department of Computer Science, Cornell University, Ithaca, NY, 1975.

[16] L. G. VALiant, The complexity of enumeration and reliability problems, this Journal, 8 (1979), pp. 410-421.

[17] R. R. WYLLIE, A theorem concerning directed graphs with applications to network reliability, Networks, 10 (1980), pp. 71-78. 